\title{
APUNTES EN PEDAGOGÍA FAMILIAR
}

\author{
Reseña a cargo de la Prof. Dra. Adriana Aristimuño \\ Decana de la Facultad de Ciencias Humanas.
}

El libro, de 95 páginas, está organizado en una introducción y 3 capítulos, y se refiere a la Pedagogía Familiar, área de las ciencias de la educación que se ocupa de los procesos educativos que tienen lugar dentro de las familias.

La obra se apoya en investigaciones de la autora, en contribuciones de memorias de grado y tesis de maestría que ella ha conducido, así como en estudios internacionales de difícil acceso y contribuciones de responsables de centros pioneros en el área, en Bélgica y Canadá.

El libro ha sido escrito para docentes, profesionales y padres, y la autora expresa que ha sido varias veces solicitado por los alumnos de sus cursos.

En el primer capítulo se profundiza en las percepciones que tienen los niños y adolescentes sobre las familias y los roles familiares. En él se reseña una investigación realizada en niños y adolescentes de 11 países de América Latina, basada en una encuesta con frases a ser completadas o respondidas con múltiple opción. Entre sus resultados destaca que siempre aparece el modelo tradicional de familia, aunque aparezcan cambios en los roles materno y paterno. Se reseña también una segunda investigación, en niños de 4 y 5 años, realizada en base a observación, dibujos por ellos realizados, y entrevistas. En esta investigación también afloran diferencias en la manera de ejercer el rol entre padres y madres. Se sintetiza una investigación sobre el rol de los abuelos, en la que se destaca la importancia de su rol en la sociedad contemporánea. Se reseña un estudio realizado en niños entre 9 y 11 años, de contexto vulnerable que asisten a comedores públicos, en el conurbano bonaerense, focalizado en la percepción que tienen sobre la familia. Por último, también se reseña un estudio realizado en niños entre 4 y 6 años, que viven situaciones de violencia, en el que se destaca la percepción negativa que los niños maltratados tienen sobre sí mismo y sobre sus padres, en relación a los que no son maltratados.

En el segundo capítulo se profundiza en el tema de las familias con niños con dificultades de aprendizaje. En él se pone énfasis en el rol que la familia puede cumplir en la detección temprana de estas dificultades. También se analizan las diferentes reacciones que tienen las familias ante las dificultades de los niños, y el impacto que esto genera (desmotivación, baja satisfacción general, reacciones inadecuadas con el niño, entre otras). Se reportan investigaciones sobre la participación de los padres en la evaluación de las dificultades de los hijos, y trabajos que se ocupan de intervenciones con padres de estos niños y jóvenes.

En el tercer capítulo se profundiza en la formación de los docentes como propuesta de optimización del vínculo escuela-familia. Se presenta abundante evidencia empírica en torno al hecho de que no existe formación sobre este tema para docentes, y se insiste en la relevancia del vínculo entre la escuela y la familia. Se citan investigaciones en torno a cómo inciden una y otra en los resultados de aprendizaje. Asimismo, se citan investigaciones sobre las representaciones que sobre la familia trasmite el sistema educativo (por ej., a través de los contenidos de los programas o de investigaciones, alguna de ellas similares a las realizadas en la Pontificia Universidad Católica del Chile). Los tres capítulos presentan bibliografía.

El libro es una muy buena síntesis del estado del arte de la disciplina, que se coloca en la frontera de las ciencias sociales, la psicología y las ciencias de la educación. Como tal, puede resultar una excelente guía orientadora para profesionales de dichas áreas de conocimiento.

Está claramente escrito y estructurado, aportando claridad conceptual y una ordenada guía de investigaciones y obras sobre el área. Es destacable el trabajo de sistematización y agrupación de trabajos por tema, dentro del amplio campo de estudio que se aborda. 
Puede constituirse también en una guía para profundizar en algunas de las subáreas identificadas, dada la amplia bibliografía que se incluye.

En este sentido, la obra cumple con creces la demanda de las estudiantes del área, constituyéndose en una obra de referencia ineludible para profesionales, en el país y la región.

González Tornaría, María del Lujan (2008). Apuntes en pedagogía familiar. Montevideo: Prensa Médica Latinoamericana. 